\title{
Refeeding Syndrome in Oncology: Report of Four Cases
}

\author{
Martin Windpessl ${ }^{\mathrm{a}, \mathrm{b}}$, Beate Mayrbaeurla ${ }^{\mathrm{a}}$, Christian Baldinger ${ }^{\mathrm{a}}$, Gernot Tiefenthaller ${ }^{\mathrm{a}}$, \\ Friedrich C. Prischla ${ }^{\mathrm{a}}$, Manfred Wallner ${ }^{\mathrm{a}}$, Josef Thaler ${ }^{\mathrm{a}}$
}

\begin{abstract}
The term refeeding syndrome (RFS) refers to the metabolic perturbations and its attendant complications in subjects who are refed after fasting. The syndrome is characterized by profound shifts of electrolytes and fluids. Its consequences are widespread and sometimes fatal. Patients with malignancies are especially vulnerable due to the presence of multiple comorbidities. We report the course of four patients with malignant or hematological disorders who developed RFS while being treated for their underlying illness. All physicians caring for susceptible patients should be cognizant of the risks of refeeding and treat RFS appropriately to reduce patient morbidity as well as mortality.
\end{abstract}

Keywords: Refeeding; Hypophosphatemia; Cancer; Oncology; Malnutrition

\section{Introduction}

Not widely known outside nutritional support circles, the term refeeding syndrome (RFS) denotes a cascade of metabolic disturbances and clinical symptoms that may develop when previously malnourished patients are refed carbohydrates, whether this is by the oral, enteral or parenteral route [1]. The syndrome is characterized by profound shifts of electrolytes and fluids, with hypophosphatemia constituting the cardinal biochemical feature. Serious cardiac, respiratory and neurologic complications may occur; in extreme cases, these complications may prove fatal [2]. This notwithstanding, protocols aimed at its prevention and management are lacking in many hospitals.

While anorexia nervosa is the modern prototypical predisposing condition, there are many clinical situations in which RFS may occur [1,3]. Cancer patients undergoing chemotherapy seem particularly vulnerable due to the presence of several comorbidities, both disease- and treatment-related $[4,5]$.

Manuscript accepted for publication February 13, 2017

aDepartment of Internal Medicine IV, Klinikum Wels-Grieskirchen, Wels, Austria

${ }^{\mathrm{b}}$ Corresponding Author: Martin Windpessl, Department of Internal Medicine IV, Klinikum Wels-Grieskirchen, Grieskirchnerstrasse 42, 4600 Wels, Austria. Email: martin.windpessl@klinikum-wegr.at

doi: https://doi.org/10.14740/wjon1007w
Therefore, RFS represents an important aspect of supportive oncology. Given its non-specific symptoms, recognizing RFS can be a difficult task. As such, it remains "underdiagnosed and undertreated" [6].

In this case series, we present the clinical course of four patients with malignant or hematological disorders who experienced RFS while being treated for their underlying illness. We aim to raise awareness of this syndrome in physicians involved in nutritional support, provide suggestions for the prevention of RFS and outline treatment strategies, according to the evidence in the literature. We believe that local guidelines should be established. Hospital nutrition teams, where available, may help in this regard.

\section{Case Reports}

\section{Case 1}

A 35-year-old woman with longstanding rheumatoid arthritis was admitted because of febrile neutropenia, presumably drugrelated. Her body mass index (BMI) was $18.3 \mathrm{~kg} / \mathrm{m}^{2}$. On examination, severe mucositis was apparent. Laboratory results showed an absolute neutropenia and a significant inflammatory response. Electrolytes and renal function were normal on admission (Table 1). A diagnosis of methotrexate-induced neutropenia was made. The patient was isolated and commenced on broad spectrum antibiotic treatment (piperacillin-tazobactam) and a granulocyte-colony stimulating factor was given. As she remained unable to eat, a central line was placed and intravenous nutrition was started at a rate of $18 \mathrm{kcal} / \mathrm{kg} / \mathrm{day}$ after 10 days of negligible food intake. B-multivitamins were added. The infusion rate was not altered, but within the next 3 days phosphate plummeted to $0.15 \mathrm{mmol} / \mathrm{L}$ (normal: $0.7-1.3$ $\mathrm{mmol} / \mathrm{L}$ ). Edema developed and the patient complained of diffuse abdominal pain. In view of her electrolyte disturbance and fluid retention, a diagnosis of RFS was made. Parenteral phosphate supplementation was prescribed. Nutrition was withheld temporarily and restarted at a slower rate. By hospital day 10, electrolytes had normalized. In parallel, the blood count had recovered and the patient was discharged after 3 weeks.

\section{Case 2}

A 69-year-old man with advanced squamous cell carcinoma of the tonsil was admitted for palliative chemotherapy. Within 
Table 1. Laboratory Findings of Four Consecutive Patients With the Refeeding Syndrome

\begin{tabular}{|c|c|c|c|c|c|c|c|c|c|c|c|c|c|c|c|c|}
\hline & \multicolumn{4}{|c|}{ Patient 1} & \multicolumn{4}{|c|}{ Patient 2} & \multicolumn{4}{|c|}{ Patient 3} & \multicolumn{4}{|c|}{ Patient 4} \\
\hline & $\mathbf{A 1}$ & B5 & C8 & D13 & A1 & B13 & C19 & D22 & A1 & B8 & C10 & D15 & A1 & B4 & $\mathrm{C} 7$ & D20 \\
\hline $\mathrm{Na}^{+}(\mathrm{mmol} / \mathrm{L})$ & 137 & 142 & 141 & 143 & 136 & 127 & 128 & 129 & 139 & 129 & 127 & 134 & 151 & 157 & 136 & 140 \\
\hline $\mathrm{Ca}^{2+}(\mathrm{mmol} / \mathrm{L})$ & 2.47 & 2.08 & 2.04 & 2.33 & 2.35 & 2.00 & 1.75 & 1.64 & 2.40 & 2.13 & 2.00 & 2.30 & 2.63 & 1.99 & 2.06 & 1.83 \\
\hline $\mathrm{Mg}^{2+}(\mathrm{mmol} / \mathrm{L})$ & 0.75 & 0.67 & 0.71 & 0.84 & 0.59 & 0.58 & 0.37 & 0.40 & 0.75 & 0.69 & 0.54 & 0.66 & 0.81 & 0.65 & 0.45 & 0.63 \\
\hline
\end{tabular}

A: admission; B: start of nutritional support; C: phosphate nadir; D: last lab result before discharge. Numbers denote hospital day.

the preceding 6 months, he had lost $30 \%$ of his usual body weight. His current BMI was $16.9 \mathrm{~kg} / \mathrm{m}^{2}$. On admission, mild hypomagnesemia was present, otherwise electrolytes were in the normal range (Table 1). A percutaneous endoscopic gastrostomy (PEG) was placed because of dysphagia. On day 5, cetuximab and docetaxel were administered as scheduled. Due to poor wound healing, the feeding tube could not be used immediately and parenteral nutrition via a pre-existing port-acath was introduced for 3 days at a daily rate of $24 \mathrm{kcal} / \mathrm{kg} /$ day. At that point, he had not eaten properly for 9 days. Subsequently, enteral nutrition was commenced via the PEG at a rate of $24 \mathrm{kcal} / \mathrm{kg} /$ day for 5 days. Profound hypophosphatemia developed (nadir $0.27 \mathrm{mmol} / \mathrm{L}$; normal: 0.7 - $1.3 \mathrm{mmol} / \mathrm{L}$ ) and hypomagnesemia and hypocalcemia worsened concurrently. Within 12 days from admission, marked fluid retention had occurred amounting to a gain of weight of $10 \mathrm{~kg}$. Nutrition was withheld intermittently, parenteral phosphate was administered and feeding was restarted at a slower rate. Except for magnesium, his electrolytes normalized within the following 4 days and he was discharged after 2 weeks.

\section{Case 3}

A 66-year-old woman was diagnosed with metastasized squamous cell cancer of the anus and admitted for initiation of palliative chemotherapy. Over the last 4 months, she had lost $12 \%$ of weight and her BMI was $20.8 \mathrm{~kg} / \mathrm{m} 2$. After implantation of a port-a-cath, 5-fluorouracil and cisplatin were administered as per protocol. Despite supportive measures, the treatment was poorly tolerated and complicated by prolonged anorexia. Due to severe gingivitis, she had barely eaten for 5 days and parenteral nutrition was prescribed at a rate of $18 \mathrm{kcal} / \mathrm{kg} /$ day and left unaltered for the following 4 days. Electrolytes showed typical features of the RFS (Table 1) and the patient complained of generalized languor. After electrolyte supplementation, salt restriction and cautious switch to oral nutrition, her symptoms slowly abated.

\section{Case 4}

Five days after his third cycle of chemotherapy (5-fluorouracil/ cisplatin) for adenocarcinoma of the esophagus, a 72-year-old man was admitted with severe mucositis. Within 3 months, he had lost $18 \%$ of his usual weight and his BMI was $21.9 \mathrm{~kg} / \mathrm{m}^{2}$ on admission. He had had negligible oral intake for 8 days. Electrolytes were normal but marked hyperglycaemia was present, deemed due to high dose dexamethasone (Table 1). Insulin sliding-scale was commenced and glucose normalized subsequently. On hospital day 4, the patient became septic and was transferred to the intensive care unit (ICU) for inotropic support and broad cover antibiotic treatment (piperacillintazobactam). Whilst on ICU, parenteral nutrition was commenced at a rate of $15 \mathrm{kcal} / \mathrm{kg} /$ day for 2 days which triggered a precipitous drop in phosphate. Parenteral phosphate substitution improved values to some extent. After 3 days, he could be transferred back to the normal ward. Parenteral nutrition was restarted at the same rate and continued unchanged for 6 days. In parallel, electrolytes decreased again. Within 1 week, he had gained $9 \mathrm{~kg}$ in weight. Edema was present. A diagnosis of RFS was made, possibly aggravated by concurrent sepsis and sodium-containing antibiotics. Electrolytes were substituted aggressively and salt and fluid restriction was implemented. The patient was finally discharged after 3 weeks with normal electrolytes.

The salient laboratory results of all cases are summarized in Table 1. The course of individual serum phosphate levels is depicted in Figure 1.

\section{Discussion}

The perils of reinstituting nutrition after periods of starvation have been recognized since World War II, with victims of famine being the first documented cases. However, it was only with the advent of widespread nutritional support that this phenomenon has garnered renewed attention. More than 30 years ago, reporting the death of two malnourished patients who were fed "overzealously", Weinsier and Krumdieck coined the term "refeeding syndrome" [7].

From a pathophysiological view, malnourished patients gradually develop a total body depletion of phosphorous. Insulin levels fall during starvation as the body switches from carbohydrate to fat metabolism. Following the delivery of glucose as part of a feeding regimen, the sudden mount in insulin secretion reverses catabolism to anabolism [8]. Due to the associated transcellular flux of electrolytes and the increased 


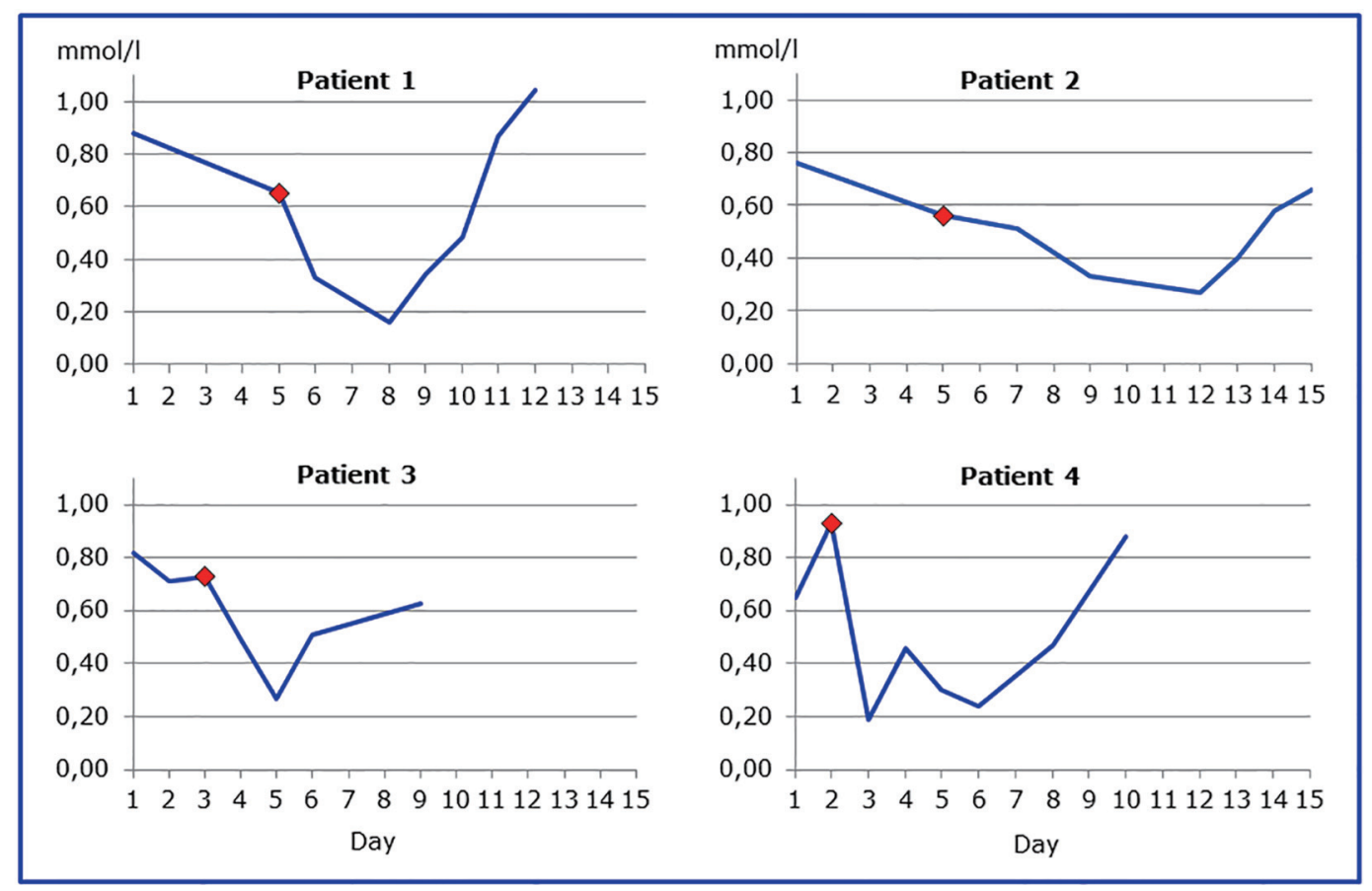

Figure 1. Courses of individual serum phosphate levels. The red diamond depicts the start of nutrition.

cellular demands, the serum concentration of these agents can fall dramatically. Hypophosphatemia, which constitutes the biochemical hallmark of RFS, is almost universally present [9]. Not surprisingly, in the treatment of diabetic ketoacidosis, analogous metabolic perturbations occur. The insulin surge also enhances fluid and sodium retention, further contributing to morbidity [10]. Concomitant thiamine deficiency can occur and may present as Wernicke encephalopathy [1].

While RFS is typically presented as a single entity, in practice it constitutes a spectrum disorder that occurs under particular circumstances within a high-risk population, i.e., malnourished patients with negligible food intake for several days at the time of refeeding. No unanimously agreed definition exists [11]. Clinical manifestations are related directly to electrolyte and vitamin deficiency but are variable and unpredictable [12]. Cardiac arrhythmias and heart failure are the most-dreaded aspects of the RFS. Fluid retention, respiratory failure and neuromuscular problems such as paralysis, seizures and rhabdomyolysis may also occur. Case reports have emphasized the potentially fatal nature of this condition $[2,7]$.

There is a paucity of data about the incidence of RFS in patients with cancer. However, in a Mexican study, it was reported to be as high as $25 \%$ [13]. Individuals with head and neck cancer (HNC) are particularly vulnerable for this phenomenon. A Danish report found an incidence of $20 \%$ in HNC patients referred for surgery $(n=54)$, with a history of previous radiation therapy among the predisposing factors [14]. Likewise, in a Dutch cohort of internal medicine patients $(n=178)$, sepsis and malignancy conferred the highest risk for the development of RFS [10]. Disease-related malnutrition reportedly occurs in approximately half of oncology patients and is associated with negative outcomes $[4,12,15]$. Pre-existing electrolyte disorders are common in these patients, whether this is paraneoplastic, associated with chemotherapeutic regimens or other com-

Table 2. Guideline for the Management of the RFS (Adapted From Refs. [5, 21, 22])

Identify patients at risk ("Discussion")

Check electrolytes prior to re-alimentation and correct deficiencies

Administer thiamine 200 - $300 \mathrm{mg}$ daily

Limit salt and fluid intake

Start feeding $10 \mathrm{kcal} / \mathrm{kg} /$ day and slowly increase over the first week

Monitor electrolytes and weight daily

If severe hypophosphatemia $(<0.3 \mathrm{mmol} / \mathrm{L})$ develops: give phosphate parenterally*

*Assuming normal renal function, parenteral phosphate repletion is generally safe with doses up to 45 mmol with infusion rates up to $20 \mathrm{mmol}$ per hour. Individualized frequent checks are recommended (e.g. after 6 and $12 \mathrm{~h}$ on day 1) [25].

For detailed recommendations regarding electrolyte substitution, see particularly Ref. [22]. 
Table 3. NICE Criteria for Identifying Patients at High Risk of Refeeding Problems (Adapted From [21])

\begin{tabular}{|c|c|c|c|c|}
\hline & 1 & 2 & 3 & 4 \\
\hline \multicolumn{5}{|l|}{ One or more of the following: } \\
\hline $\mathrm{BMI}<16 \mathrm{~kg} / \mathrm{m}^{2}$ & - & - & - & - \\
\hline Weight loss $>15 \%$ within the last 3 - 6 months & $\mathrm{n} / \mathrm{a}$ & + & - & + \\
\hline Little or no nutritional intake for more than 10 days & + & - & - & - \\
\hline Low levels of electrolytes prior to feeding & + & + & + & + \\
\hline \multicolumn{5}{|l|}{ Two or more of the following: } \\
\hline $\mathrm{BMI}<18.5 \mathrm{~kg} / \mathrm{m}^{2}$ & + & + & - & - \\
\hline Weight loss $>10 \%$ within the last $3-6$ months & $\mathrm{n} / \mathrm{a}$ & + & + & + \\
\hline Little or no nutritional intake for more than 5 days & + & + & + & + \\
\hline History of alcohol abuse or drugs including chemotherapy & - & + & + & + \\
\hline
\end{tabular}

Numbers 1 - 4: individual patients. +/- denotes presence/absence of a risk factor. n/a: not available.

pounds frequently used in this clinical setting, as exemplified by case $2[16,17]$. Hypophosphatemia can be frequently found in patients with sepsis, especially in Gram-negative bacteremia and its extent correlates with illness severity [18]. Electrolyte disorders such as hypokalemia can also arise as a consequence of antibiotic therapy. Moreover, sodium-containing antibiotic regimens such as piperacillin-tazobactam can lead to fluid retention [19]. Therefore, it is often difficult if not impossible to ascribe biochemical and clinical complications to one of several causative factors. As illustrated by case 4, these events often occur in parallel and should be seen as potentiating each other rather than causing the RFS singularly.

Timely recognition of patients at risk is crucial before initiating nutritional support. Any patients with negligible food intake for more than 5 days should be deemed at risk of developing refeeding problems [20]. The short nutritional assessment questionnaire (SNAQ) had a high negative predictive value for the diagnosis of RFS in one observational study [10]. In 2006, the British National Institute for Clinical Excellence (NICE) came up with criteria for identifying patients at risk and offered treatment strategies and is still considered a useful tool in daily practice $[10,21]$. Of note, a history of recent chemotherapy is one of several risk factors cited for RFS. In 2013, the Irish Society for Clinical Nutrition \& Metabolism followed suit with detailed instructions for electrolyte replacement [22]. Both guidelines could serve as templates for local protocols as shown in Table $2[5,21,22]$. By using these criteria, all patients presented could have been found to be at high risk of RFS before the start of nutrition (Table 3) [21]. At first glance, refeeding was started rather carefully, with only $1,000 \mathrm{kcal} /$ day (ranging from 15 to $24 \mathrm{kcal} / \mathrm{kg} /$ day in the respective cases) being delivered. However, in such high-risk patients, starting nutrition support at a maximum of $10 \mathrm{kcal} / \mathrm{kg} /$ day is recommended, increasing levels slowly to meet full needs within 1 week [21].

Prior to refeeding, electrolytes should be checked. Thiamine is recommended upfront realimentation in at-risk patients. Gradual introduction of calories particularly over the first week of refeeding is prudent until the patient is metabolically stable ("start low and go slow"). Initially, fluid and salt intake should be conservative because of the propensity to retain these [23]. As noted, attention needs to be paid to biochemical indices, and electrolytes should be measured daily. Once overt RFS has developed, caloric intake should be reduced, electrolyte abnormalities treated aggressively and extra $\mathrm{B}$ vitamins given.

Despite considerable progress in our understanding of its pathophysiology over the last decades, RFS remains underdiagnosed, causing avoidable morbidity in core patient groups at risk. The present case series illustrates that cancer patients are particularly prone for this phenomenon. Because disease- and treatment-related complications such as electrolyte disturbances and infections often occur in parallel, diagnosis of RFS can be delayed. Moreover, it is not unusual for chemistry panels not to include phosphate unless specifically ordered and it is partly therefore that phosphate has been dubbed a "neglected electrolyte" [24]. Our experience emphasizes the need for better recognition and monitoring.

To summarize, clinical diagnosis of RFS requires vigilance. As stated by Marinella, physicians involved with providing supportive care to cancer patients ought to be familiar with this syndrome and its attendant complications to prevent morbidity and mortality [25].

\section{Conflicts of Interest}

The authors have no conflicts of interest.

\section{References}

1. Crook MA, Hally V, Panteli JV. The importance of the refeeding syndrome. Nutrition. 2001;17(7-8):632-637.

2. Boateng AA, Sriram K, Meguid MM, Crook M. Refeeding syndrome: treatment considerations based on collective analysis of literature case reports. Nutrition. 2010;26(2):156-167.

3. Mehanna HM, Moledina J, Travis J. Refeeding syndrome: what it is, and how to prevent and treat it. BMJ. 2008;336(7659):1495-1498. 
4. Marinella MA. Refeeding syndrome: an important aspect of supportive oncology. J Support Oncol. 2009;7(1):1116.

5. Stanga Z, Brunner A, Leuenberger M, Grimble RF, Shenkin A, Allison SP, Lobo DN. Nutrition in clinical practice-the refeeding syndrome: illustrative cases and guidelines for prevention and treatment. Eur J Clin Nutr. 2008;62(6):687-694.

6. Gariballa S. Refeeding syndrome: a potentially fatal condition but remains underdiagnosed and undertreated. Nutrition. 2008;24(6):604-606.

7. Weinsier RL, Krumdieck CL. Death resulting from overzealous total parenteral nutrition: the refeeding syndrome revisited. Am J Clin Nutr. 1981;34(3):393-399.

8. Walmsley RS. Refeeding syndrome: screening, incidence, and treatment during parenteral nutrition. J Gastroenterol Hepatol. 2013;28(Suppl 4):113-117.

9. Kagansky N, Levy S, Koren-Morag N, Berger D, Knobler H. Hypophosphataemia in old patients is associated with the refeeding syndrome and reduced survival. J Intern Med. 2005;257(5):461-468.

10. Kraaijenbrink BV, Lambers WM, Mathus-Vliegen EM, Siegert CE. Incidence of refeeding syndrome in internal medicine patients. Neth J Med. 2016;74(3):116-121.

11. Miller SJ. Death resulting from overzealous total parenteral nutrition: the refeeding syndrome revisited. Nutr Clin Pract. 2008;23(2):166-171.

12. Palesty JA, Dudrick SJ. Cachexia, malnutrition, the refeeding syndrome, and lessons from Goldilocks. Surg Clin North Am. 2011;91(3):653-673.

13. Gonzalez Avila G, Fajardo Rodriguez A, Gonzalez Figueroa E. [The incidence of the refeeding syndrome in cancer patients who receive artificial nutritional treatment]. Nutr Hosp. 1996;11(2):98-101.

14. Rasmussen SO, Kristensen MB, Wessel I, Andersen JR. Incidence and Risk Factors of Refeeding Syndrome in Head and Neck Cancer Patients-An Observational Study.
Nutr Cancer. 2016;68(8):1320-1329.

15. Vandebroek AJ, Schrijvers D. Nutritional issues in anticancer treatment. Ann Oncol. 2008;19(Suppl 5):v52-55.

16. Rosner MH, Dalkin AC. Electrolyte disorders associated with cancer. Adv Chronic Kidney Dis. 2014;21(1):7-17.

17. Miltiadous G, Christidis D, Kalogirou M, Elisaf M. Causes and mechanisms of acid-base and electrolyte abnormalities in cancer patients. Eur J Intern Med. 2008;19(1):1-7.

18. Geerse DA, Bindels AJ, Kuiper MA, Roos AN, Spronk PE, Schultz MJ. Approach to hypophosphataemia in intensive care units - a nationwide survey. Neth J Med. 2012;70(9):425-430.

19. Zietse R, Zoutendijk R, Hoorn EJ. Fluid, electrolyte and acid-base disorders associated with antibiotic therapy. Nat Rev Nephrol. 2009;5(4):193-202.

20. Crook MA. Refeeding syndrome: problems with definition and management. Nutrition. 2014;30(11-12):14481455.

21. National Institute for Clinical Excellence. Nutrition Support for Adults. Clinical Guideline CG32. 2006 Feb 1; 1-176. Cited December 2016. Available from URL: http:// www.nice.org.uk/nicemedia/live/10978/29981/29981. pdf.

22. Irish Society for Clinical Nutrition \& Metabolism. Prevention and Treatment of Refeeding Syndrome in the Acute Care Setting. IrSPEN Guideline 1. November 2013; 1-31. Cited December 2016. Available from URL: http:/www.irspen.ie/wp-content/uploads/2013/12/Refeeding-Syndome-Guideline-Doc.pdf.

23. Brooks MJ, Melnik G. The refeeding syndrome: an approach to understanding its complications and preventing its occurrence. Pharmacotherapy. 1995;15(6):713-726.

24. Workman ML. Magnesium and phosphorus: the neglected electrolytes. AACN Clin Issues Crit Care Nurs. 1992;3(3):655-663.

25. Marinella MA. Refeeding syndrome in cancer patients. Int J Clin Pract. 2008;62(3):460-465. 